\title{
Free-standing conductive thin films from gold-carbon nanotube composites
}

\author{
Dawid Janas $^{\mathrm{a}, \mathrm{b},{ }^{*}, \text { Monika Rdest }^{\mathrm{a}} \text {, Krzysztof K.K. Koziol }}{ }^{\mathrm{a}}$ \\ ${ }^{a}$ Department of Materials Science and Metallurgy, University of Cambridge, 27 Charles \\ Babbage Rd, CB3 OFS Cambridge, United Kingdom \\ ${ }^{b}$ Department of Chemistry, Silesian University of Technology, B. Krzywoustego 4, 44-100 \\ Gliwice, Poland
}

\begin{abstract}
We report on the development of a method of formation of free-standing gold-carbon nanotube (CNT) composites. It is based on a quick and simple decoration of CNT films with the application of chloroauric aqueous solution. Flexible and conductive macroassemblies of any size and shape can be created with ease. Addition of as little as $1 \%$ of Au by weight allowed for reduction of resistance by more than a quarter. Higher Au doping resulted in further decrease of resistance, but not so significant. From the practical point of view, these composites are a new type of materials with high electrical conductivity, but also offer resistance to corrosion.
\end{abstract}

Key words: carbon nanotubes; macroassemblies; gold nanoparticles

\section{Introduction}

Carbon nanomaterials such as carbon nanotubes (CNTs) and graphene have shown great promise for creation of materials of significantly improved characteristics as compared with the typical materials present around us from hundreds of years. High performance in terms of electrical [1, 2], thermal [3-5], mechanical $[6,7]$ and optical $[8,9]$ properties give us hope that we will be able to make full use of them to offer significant improvements on many fronts one day. However, the problem is in translation these encouraging properties from individual CNTs and graphene flakes to the macroscopic objects. Their macroscopic assemblies often have a sort of discontinuous nature with plethora of voids, which disrupts transport of phonons/electrons

\footnotetext{
* Corresponding author. Tel/Fax: +48 32 2372958. E-mail address: dawid.janas@polsl.pl (D. Janas).
} 
and load transfer. R\&D community needs to either to reduce this number of voids by creating much longer CNTs/larger graphene flakes or find appropriate "glue", to combine these individual elements without significant deterioration of their properties.

Deposition of Au onto CNTs can be accomplished by multi-step electroless liquid processing [10-12], electrodeposition [13] or physical methods such as sputtering $[14,15]$ or electron-beam evaporation [16]. One of the most facile approach is based on the use of $\mathrm{HAuCl}_{4}$ aqueous solution [17, 18], but it commonly uses CNT powder as "feed". The technique has not been tested before on CNT macroassemblies such as free-standing thin films of tangible dimensions, what is crucial to evaluate their properties and practical potential.

Here we present a convenient method of manufacturing mechanically-strong free-standing AuCNT composites of any size and shape. Au clusters improve electron transport between individual CNTs and their bundles, which results in increased electrical conductivity. The material offers high corrosion resistance compared to traditional metal conductors or CNTs typically doped with metals such as $\mathrm{Cu}$ or $\mathrm{Al}$.

\section{Experimental}

The CNT films were produced by a method reported elsewhere [19]. In brief, CNTs were combined with a binder (ethyl cellulose), dispersed and spray-coated onto a substrate, from which it can be delaminated as free-standing CNT films. Then, flash annealing with a blowtorch rapidly removes the binder making free of residue CNT film. For this study we employed both the CNT films with the binder (b) and binder-free (bf) to see the influence of its composition on deposition of Au. They are denoted as CNT-b and CNT-bf, respectively.

A gold ion solution was prepared by adding chloroauric acid ( $\mathrm{HAuCl}_{4}$, Sigma Aldrich, $\left.99.9 \%\right)$ to deionized water, sonicated for 5 minutes, dripped onto the CNT films to fully cover their surface, and then left to dry in the ambient conditions. Equal volumes for deposition of $1 \%$ wt and 5\%wt aqueous solutions were used. 
Scanning Electron Microscopy (SEM, FEI Nova NanoSEM) and Transmission Electron Microscopy (TEM, Tecnai Osiris) was used to probe the structure and quality of CNTs films on micro- and nanoscale.

Energy-Dispersive X-ray spectroscopy (EDX, Bruker Quantax coupled with Nova NanoSEM) detected the elemental composition at $10 \mathrm{keV}$ emission voltage.

Thermogravimetric analysis (TGA, Mettler Toledo TGA/DSC system) measured changes in chemical and physical properties with temperature. The samples were heated to $1000^{\circ} \mathrm{C}$ at $10^{\circ} \mathrm{C}$ $\min ^{-1}$ rate in the flow of air at $20 \mathrm{ml} \mathrm{min}{ }^{-1}$.

Electrical properties measurements were performed with the use of Keithley 2000 multimeter using a four probe method. $5 \mathrm{~mm}$ x $40 \mathrm{~mm}$ specimens were prepared for this purpose. An average and standard deviation among ten samples for each subset (CNT-b and CNT-bf subjected to $0 \% \mathrm{wt}, 1 \% \mathrm{wt}, 5 \% \mathrm{wt}$ Au deposition) is given. Resistance was normalized to the measured initial resistance recorded at room temperature.

\section{Results}

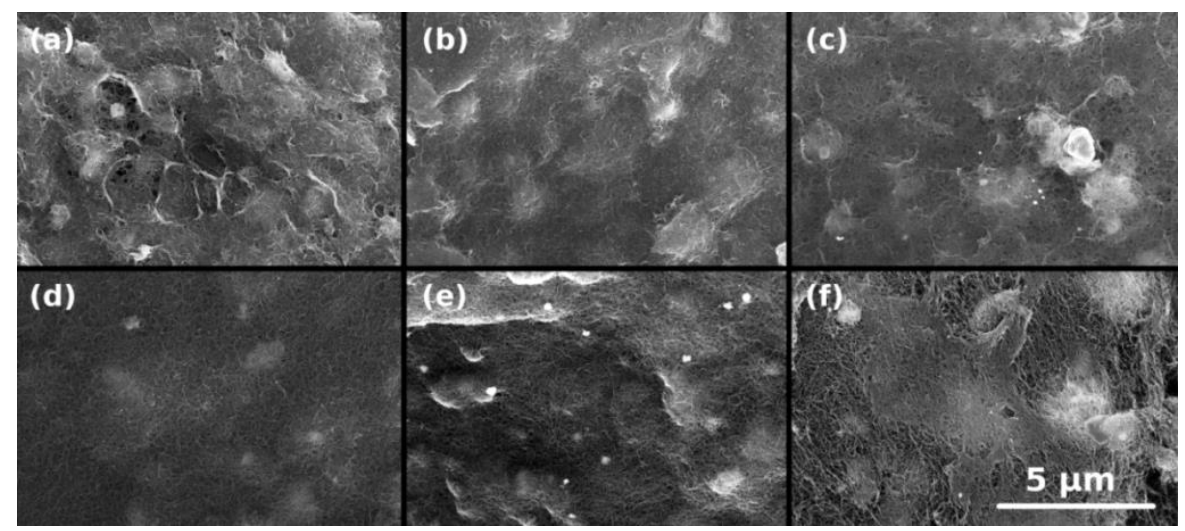

Fig. 1 SEM micrographs of the CNT films. (a) CNT-b (b) CNT-b with 1\%wt Au deposition, (c) CNT-b with 5\%wt Au deposition, (d) CNT-bf, (e) CNT-bf with 1\%wt Au deposition, (f) CNTbf with 5\%wt Au deposition

Two types of films were used for Au deposition: CNT-b (with the binder) and CNT-bf (binderfree). Fig. 1 shows microstructure of both of them with and without Au present. It is not 
surprising that the CNT-bf is more porous because of the absence of ethyl cellulose, which created a sort of removable scaffolding for the CNT film formation. In fact, Au deposition seems to gradually increase the porosity of the binder-free material (Fig. 1d-f). Furthermore, deposition of $\mathrm{Au}$ appears to be uniform without excessive agglomeration of clusters. Because the process forms mostly $\mathrm{Au}$ in the nanoscale range [18], metal particles are not visible by SEM. Highresolution TEM (Fig. 2) showed that Au clusters size range from a few up to about $20 \mathrm{~nm}$.

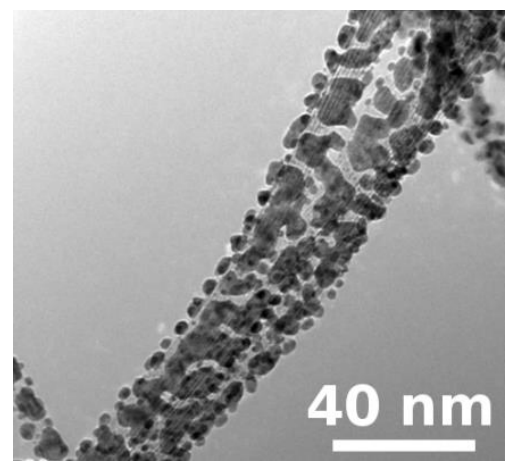

Fig. 2 TEM micrographs of the CNT-bf with 5\%wt Au deposition.

Furthermore, we carried out TGA to quantify the amount of Au deposited onto the surface. The samples in the left column, which represent the CNT-b materials with binder present, show typical, two-step influence of the oxidation of ethyl cellulose below $450^{\circ} \mathrm{C}$ [19]. This region coincides with the decomposition regime of Au compounds introduced using this method. Sideproducts of reaction between $\mathrm{HAuCl}_{4}$ and CNTs or various carbonaceous impurities commonly contaminating the sample are responsible for that low-temperature monotonic weight loss. Chlorine present in up to few wt\% content (Fig. 4a) can also react with various functional groups making them volatile [20]. If we take into account that the CNT films produced this method using unpurified CNTs contain 6.3\% (Fig. 3a, CNT-b) and 12.2\% (Fig. 3d, CNT-bf) residual alumina catalyst, we can evaluate how Au addition scales with concentration of the doping solution. In the case of CNT-bf films, we see that Au content after TGA is about $8.5 \%$ (Fig. 3e, 1\%wt Au solution) and 33.2\% (Fig. 3f, 5\%wt Au solution was used). 
(a)

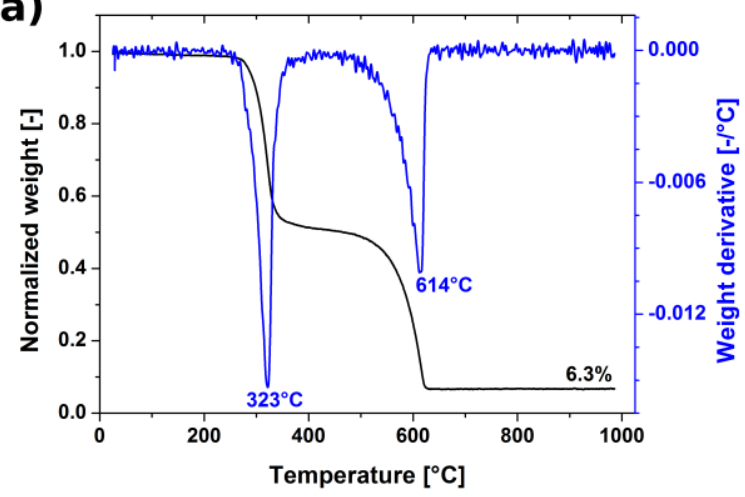

(d)

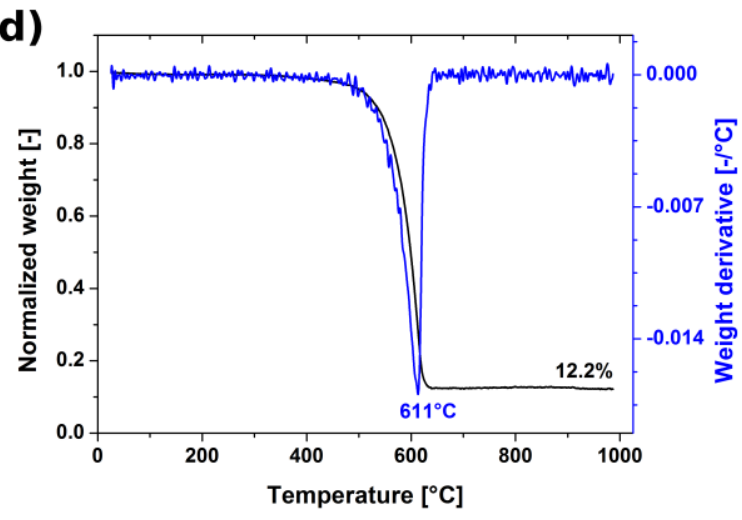

(b)

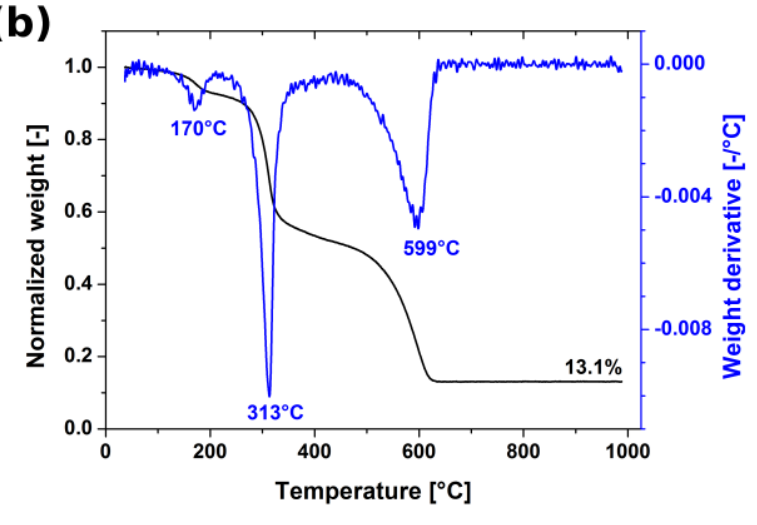

(e)

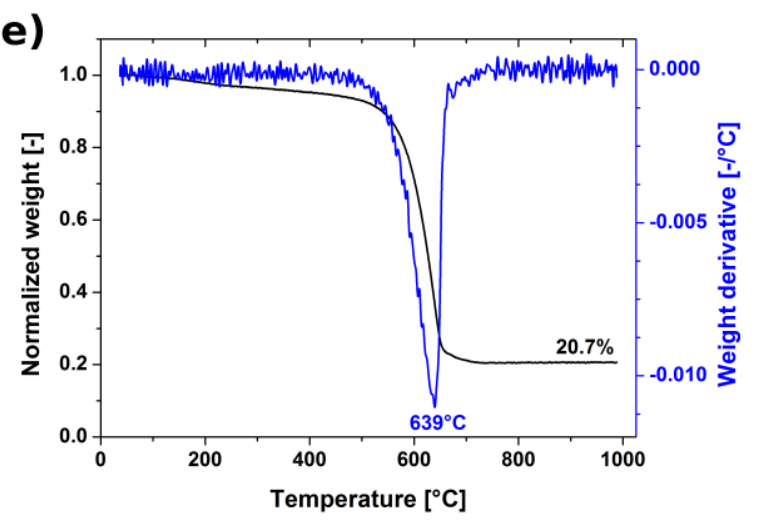

(f)

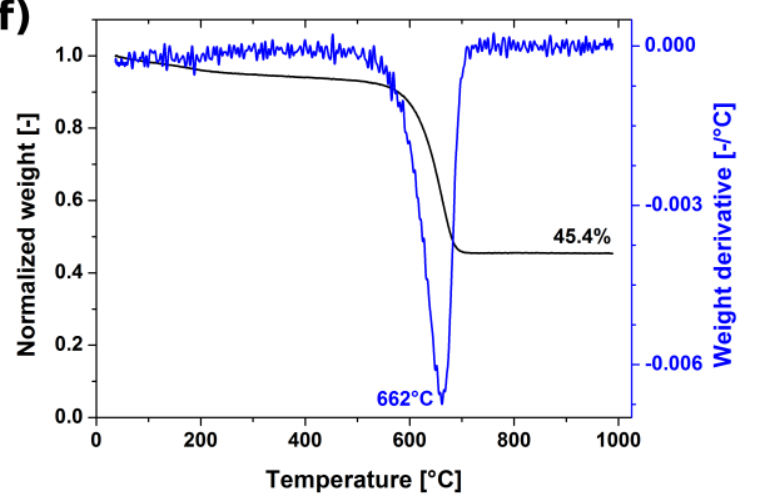

Fig. 3 Thermograms of the CNT films. (a) CNT-b (b) CNT-b with 1\%wt Au deposition, (c) CNT-b with 5\%wt Au deposition, (d) CNT-bf, (e) CNT-bf with 1\%wt Au deposition, (f) CNTbf with 5\%wt Au deposition

When CNT-b films were used, the Au content after TGA was 6.8\% (Fig. 3b, 1\%wt Au solution) and $27.7 \%$ (Fig. 3c, 5\%wt Au solution). In both cases, we expected a fifth-fold increase, whereas the results show that they quadrupled. The reason for that may be that $\mathrm{HAuCl}_{4}$ that we 
bought was not fully anhydrous and water weight loss contributed to the recorded thermograms. The explanation is supported by the fact that it was a consistent error in all of our experiments.

(a)

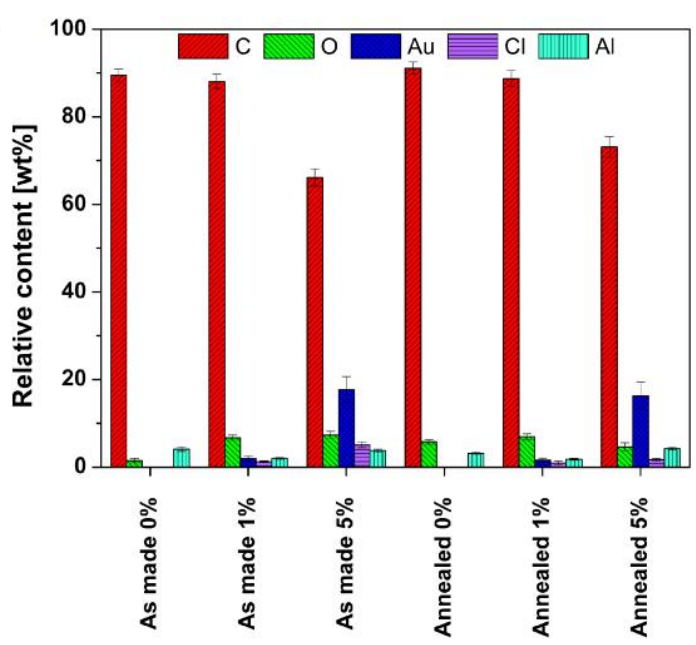

(b)

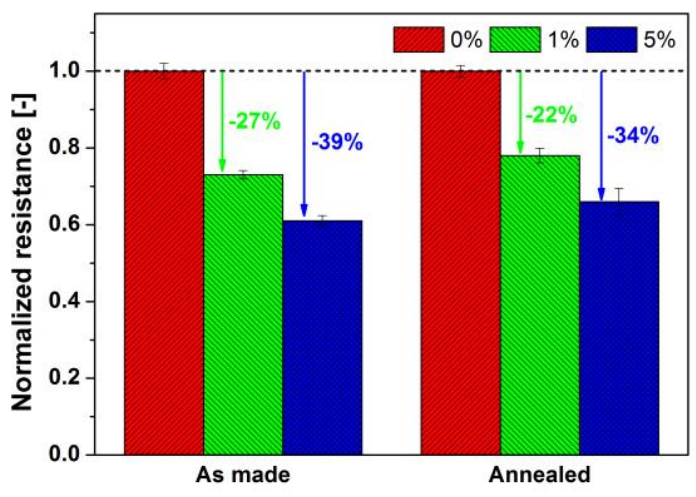

Fig. 4. (a) chemical composition of all samples as evaluated by EDX, (b) the influence of Au addition on electrical resistance of CNT films.

As a final step, we evaluated the influence of Au addition on electrical properties of CNT films (Fig. 4b). Even as small addition as that from $1 \%$ wt Au solution causes reduction in electrical resistance by a quarter. What is interesting is that further $\mathrm{Au}$ addition does not cause proportional decrease of resistance. The results show that small Au content helps with electrical percolation of the material by alleviating the problem of junction resistance. Lekawa-Raus et al. showed in a similar study that Ag nanoparticles can effectively join CNTs and their bundles to improve the electrical properties [21]. Addition of $\mathrm{Au}$ instead of $\mathrm{Ag}$ is important for long term 
performance because $\mathrm{Ag}$ is prone to oxidation, but $\mathrm{Au} / \mathrm{CNT}$ composite can preserve the corrosion-resistant nature of CNTs. From the electrical point of view, their conductivity is of the same order of magnitude $6.30 \times 10^{7} \mathrm{~S} / \mathrm{m}(\mathrm{Ag})$ vs. $4.10 \times 10^{7} \mathrm{~S} / \mathrm{m}(\mathrm{Au})[22]$.

\section{Conclusions}

In brief, we proposed a simple route towards Au/CNT composite films of any size or shape. Doping of the material is very-straightforward and the process is based on a single step, which involves putting $\mathrm{CNT}$ film in contact with the $\mathrm{HAuCl}_{4}$ aqueous solution and leaving it to dry. By varying the concentration of the solution, one can tune the content of $\mathrm{Au}$ on the surface of the material. The presence of binder, removal of which does not cause significant improve in conductivity, did not interfere with the doping efficiency despite improved packing of the material. For some applications in which only electrical conductivity is the target, leaving the film with the binder has no influence, but assures better mechanical properties. We believe that such a simple method to create free-standing Au/CNT composites could be helpful in the reallife application of CNT macroassemblies wherein high electrical conductivity is important. It is important to stress that $\mathrm{Au}$ nanoparticles are not prone to oxidation as commonly used $\mathrm{Cu}, \mathrm{Al}$ and $\mathrm{Ag}$ nanoparticles, therefore such $\mathrm{Au} / \mathrm{CNT}$ composites can be useful in the applications where corrosion-resistance is of the essence.

\section{Acknowledgements}

M.R. and K.K. acknowledge the European Research Council (under the Horizon 2020 Program, ERC grant agreement 641382) and the Royal Society for the financial support. D.J. would also like to thank National Science Center, Poland (under the Polonez program, grant agreement UMO-2015/19/P/ST5/03799) and the European Union's Horizon 2020 research and innovation programme (Marie Skłodowska-Curie grant agreement 665778). 


\section{References}

[1] S. Hong, S. Myung, Nanotube Electronics: A flexible approach to mobility, Nat Nano 2(4) (2007) 207-208.

[2] S. Stankovich, D.A. Dikin, G.H.B. Dommett, K.M. Kohlhaas, E.J. Zimney, E.A. Stach, R.D. Piner, S.T. Nguyen, R.S. Ruoff, Graphene-based composite materials, Nature 442(7100) (2006) 282-286.

[3] E. Pop, D. Mann, Q. Wang, K. Goodson, H. Dai, Thermal Conductance of an Individual Single-Wall Carbon Nanotube above Room Temperature, Nano Letters 6(1) (2006) 96-100.

[4] E.T. Thostenson, C. Li, T.-W. Chou, Nanocomposites in context, Composites Science and Technology 65(3-4) (2005) 491-516.

[5] A.A. Balandin, S. Ghosh, W. Bao, I. Calizo, D. Teweldebrhan, F. Miao, C.N. Lau, Superior Thermal Conductivity of Single-Layer Graphene, Nano Letters 8(3) (2008) 902-907.

[6] M.-F. Yu, O. Lourie, M.J. Dyer, K. Moloni, T.F. Kelly, R.S. Ruoff, Strength and Breaking Mechanism of Multiwalled Carbon Nanotubes Under Tensile Load, Science 287(5453) (2000) 637-640.

[7] C. Lee, X. Wei, J.W. Kysar, J. Hone, Measurement of the Elastic Properties and Intrinsic Strength of Monolayer Graphene, Science 321(5887) (2008) 385.

[8] D. Janas, N. Czechowski, B. Krajnik, S. Mackowski, K.K. Koziol, Electroluminescence from carbon nanotube films resistively heated in air, Applied Physics Letters 102(18) (2013) 181104.

[9] J. Liu, A.R. Wright, C. Zhang, Z. Ma, Strong terahertz conductance of graphene nanoribbons under a magnetic field, Applied Physics Letters 93(4) (2008) 041106.

[10] N.A. Kumar, S.H. Kim, J.S. Kim, J.T. Kim, Y.T. Jeong, FUNCTIONALIZATION OF MULTIWALLED CARBON NANOTUBES WITH CYSTEAMINE FOR THE CONSTRUCTION OF CNT/GOLD NANOPARTICLE HYBRID NANOSTRUCTURES, Surface Review and Letters 16(03) (2009) 487-492.

[11] Z. Lin, L. Huang, Y. Liu, J.-M. Lin, Y. Chi, G. Chen, Electrochemiluminescent biosensor based on multi-wall carbon nanotube/nano-Au modified electrode, Electrochemistry Communications 10(11) (2008) 1708-1711.

[12] M.M. Oliveira, A.J.G. Zarbin, Carbon Nanotubes Decorated with both Gold Nanoparticles and Polythiophene, The Journal of Physical Chemistry C 112(48) (2008) 18783-18786.

[13] B.M. Quinn, C. Dekker, S.G. Lemay, Electrodeposition of Noble Metal Nanoparticles on Carbon Nanotubes, Journal of the American Chemical Society 127(17) (2005) 6146-6147.

[14] D. Janas, K.K.K. Koziol, The influence of metal nanoparticles on electrical properties of carbon nanotubes, Applied Surface Science 376 (2016) 74-78.

[15] C. Muratore, A.N. Reed, J.E. Bultman, S. Ganguli, B.A. Cola, A.A. Voevodin, Nanoparticle decoration of carbon nanotubes by sputtering, Carbon 57 (2013) 274-281.

[16] Y. Zhang, N.W. Franklin, R.J. Chen, H. Dai, Metal coating on suspended carbon nanotubes and its implication to metal-tube interaction, Chemical Physics Letters 331(1) (2000) 35-41.

[17] K. Choi, C. Yu, Highly Doped Carbon Nanotubes with Gold Nanoparticles and Their Influence on Electrical Conductivity and Thermopower of Nanocomposites, PLoS ONE 7(9) (2012) e44977.

[18] E. Gravel, S. Foillard, H. Zhang, H. Li, E. Doris, Chitosan-mediated synthesis of carbon nanotubegold nanohybrids, Science China Chemistry 53(9) (2010) 2015-2018.

[19] D. Janas, M. Rdest, K. Koziol, Free-standing films from chirality-controlled carbon nanotubes, Materials \& Design (in press) (2017).

[20] D. Janas, S. Boncel, K.K.K. Koziol, Electrothermal halogenation of carbon nanotube films, Carbon 73 (2014) 259-266.

[21] A. Lekawa-Raus, P. Haladyj, K. Koziol, Carbon nanotube fiber-silver hybrid electrical conductors, Materials Letters 133 (2014) 186-189.

[22] R.A. Serway, Principles of Physics, 2nd ed., London: Saunders College Pub, Fort Worth, Texas, 1998, p. 602. 